\title{
Incidence and Pattern of Dysrhythmia in Acute Coronary Syndrome at Suez Canal University Hospital
}

\author{
Moamin A. Alassouli ${ }^{*}$ Ahmad A. El-hawary, Hanan M. Kamal, Hussein M. \\ Mahmoud
}

Department of Cardiology, Faculty of Medicine, Suez Canal University, Egypt

\begin{abstract}
Background: The incidence of arrhythmic complications in Egyptian patients with acute coronary syndromes (ACS) has not been previously reported. The present study results will serve as the local database for future studies. Aim: To evaluate the incidence of arrhythmic complications in ASC among Egyptian patients and to identify factors that may affect arrhythmia complications in ACS patients. Material and Method: Data collected from 400 patients admitted to cardiology department of Suez Canal University Hospital from 1 January 2009 to 31 December 2011, who were diagnosed as having acute coronary syndrome. This registry includes patients who presented with ACS including ST elevation myocardial infarction (STEMI), non-ST elevation myocardial infarction (NSTEMI) and unstable angina (UA). Results: The study showed that the majority of patients $(79.3 \%)$ were males of mean age $54.5+10$ years, and $70.9 \%$ were STEMI. The highest morbidity and mortality were noticed in those of STEMI $25.6 \%$ for morbidity and $4.4 \%$ for mortality , and those of anterior myocardial involvement 77.7 for morbidity and $56 \%$ for mortality. Arrhythmia was present in $74.6 \%$ of study population, $57.6 \%$ of them were tachyarrythmia, while $26.4 \%$ were bradyarrhytmia. The incidence of VT was higher in the younger age group, while AV block and arrhythmic death were higher in the older aged patients. The incidence of different types of arrhythmia was as follows: PVC (39.5\%), PAE (19.5\%), AF (16.8\%), HB (10.1\%), VT (9.6\%), arrest (asystole) (8.5\%), VF (8.1\%), RBBB (6.3\%), LBBB (4.3\%) and Nodal rhythm (3.1\%). Our demographic characteristics, risk factors for both ACS and those who had arrhythmias were the same. The patients were younger, smokers, suffering mainly of hypertension, they were more prone to arrhythmias incidence, because they were smokers, mostly STEMI, and of anterior myocardial involvement. Conclusion: Arrhythmias complicating ACS are associated with higher in-hospital mortality. This study showed that the current management strategy is so adherent to guidelines, but deficient for proper arrhythmias management.
\end{abstract}

Keywords: Cardiac arrhythmia, Ventricular tachycardia, AV block

\section{Introduction}

Patients with acute coronary syndrome (ACS) include those whose clinical presentations cover the following range of diagnoses: unstable angina, non-ST elevation myocardial infarction (NSTEMI), and ST-elevation my ocardial infarction (STEMI). This ACS spectrum concept is a useful framework for developing therapeutic strategies ${ }^{(1)}$. Cardiac arrhythmias are not uncommon during and immediately after an acute myocardial infarction (AMI). Of all patients who have an AMI, about $90 \%$ develop some form of cardiac ar- 
rhythmia. In $25 \%$ of patients, such rhythm abnormalities manifest within the first 24 hours. In this group of patients, the risk of serious arrhythmias, such as ventricular fibrillation, is greatest in the first hour and declines thereafter. The incidence increases with an STelevation myocardial infarction (STEMI) and decreases with a non ST elevation myocardial infarction (NSTEMI) ${ }^{(2)}$. In spite of significant reduction in total mortality observed in patients discharged after an acute myocardial infarction (MI), ventricular arrhythmias still account for $30-40 \%$ of deaths. This figure, which was initially provided by studies carried out in the pre-thrombolytic era, has been subsequently confirmed when most of the patients had been revascularized by thrombolysis or percutaneous coronary intervention $(\mathrm{PCl})^{(3)}$. Early revascularization and more generalized use of beta blockers, angiotensin converting enzyme inhibitors, statins, and antiplatelet agents have largely contributed to the improvement in the prognosis of patients presenting with an ST elevation in acute MI. Nevertheless, identification of patients at risk remains an issue far from being adequately addressed. There is a general consensus that depressed ventricular function, as reflected by LVEF $<40 \%$, represents the strongest negative prognostic factor in these patients $^{(4)}$.

\section{Patients and Methods}

This retrospective study was conducted on 400 patients who were admitted to the Cardiology department of the Suez Canal University Hospital with the diagnosis of acute coronary syndrome from January 2009 to December 2011. Patients with stable angina, congenital heart disease and valvular heart disease were excluded. Data of patients with ACS drawn from archival records of coronary care unit (CCU) and emergency room (ER) admissions were subjected to Full history and examination with special emphasis on the coronary risk factors, specially, age sex, obesity, smoking, other special habits of medical importance, DM, Hypertension, positive family history, previous history of prior episodes of myocardial ischemia such as, angina, myocardial infarction, $\mathrm{PCl}$, or coronary angiography, CABG, anti-ischemic treatment, and previous ER/CCU admission. 12 lead ECG of all patients were revised for ST deviation and pathological q waves; Each ECG was analyzed systematically for Rate and rhythm regularity of the heart, QRS complex morphology and width, atrioventricular $(A-V)$ relationship. Data from transthoracic echocardiography if was performed, the cardiac function by EF\%, segmental wall motion abnormalities, valvular affection or any mechanical shunts were reported. Cardiac enzymes (CPK, CPK$M B$,Troponin) and Routine labs i.e. renal functions, $C B C$ and electrolytes was taken for each patients.

In hospital course:

Morbidity defined as complications that were developed to the patients by further persistent ischemia, persistent arrhythmia, and organ failure like ventricular dysfunction, ischemic mitral regurgitation, and renal impairments. Routine (immediate) antiischemic treatment: aspirin, Clopidogrel, low-molecular-weight heparin (LMWH), Nitroglycerine, B-blocker, ACE inhibitors, Lipid lowering agents (Statin). Management of arrhythmia: electrolytes correction, antiarrhythmic drugs as Amiodarone, Lidocaine, or/and cardiovaresion (DC) shock.

\section{Statistical Analysis}

All demographic, clinical, and technical data obtained from all patients were statistically analyzed by using: SPSS program version 6 through its descriptive methods and cross tabbing for comparing. Chi-square and $p$ value for defining significance and relation 
strength of the different variables. Excel office 97 and 93 for demonstration charts.

\section{Results}

The study population included 400 patients who were admitted to the Cardiology department of Suez Canal University Hospital from 1 January 2009 to 31 December 2011, who were diagnosed as having acute coronary syndrome.The registry was a record based clinical retrospective observational study involving patients in the emergency department, and cardiac intensive care unit.

\section{Risk factors of ACS}

Most of the patients were male gender 79.2\% (316 patients), While female gender constituted $20.8 \%$ ( 83 patients). The age of patients enrolled in this study ranged from 19 years to 88 years with a mean age of $54.5 \pm 10$ years. In this study several risk factors were detected. Their order of prevalence was as follows: Smoking was the most significant risk factor among patients presented with ACS, 69.8\% (279 patients) were current smokers. Hypertension was present in $40.8 \%$ (163 patients); $39 \%$ of them were on $B B$ and $24.9 \%$ of them were on ACE inhibitors. Diabetes mellitus was present in $38.9 \%$., (123 patients) of the study population. $26.9 \%$ of them were on OHG while patients on Insulin were $15 \%$ of the study patients. Obesity was found only in $24.4 \%$ (97 patients) of the study population. Alcohol intake was present in only $2.2 \%$ i.e. 8 patients of the study patients. Others which included Hashish, or drug abuse were present in $16.3 \%$ (65 patients) of the study population.

Family history of coronary artery disease was positive in $14.6 \%$ (58 patients) of the study population. Past history of previous similar condition of ACS was present in 36.2\% (145 patients).Anti-ischemic treatment intake was present in $32.8 \%$ (131 patients). Ischemic heart disease was present in 29.2\% (117 patients). Coronary angiography was previously done in $23.4 \%$ (94 patients). CABG was previously performed in $5 \%$ (20 patients) of the study population.

Table 1: Echocardiographic findings in ACS study patients

\begin{tabular}{|l|cc|}
\hline Finding & Number of patients & $\%$ \\
\hline Normal Findings & 60 & 29.9 \\
SWMA & 132 & 65.7 \\
Impaired Systolic Function $(\mathrm{EF} \%<40 \%)$ & 60 & 29.8 \\
LV diastolic dysfunction & 117 & 58.2 \\
LV Thrombus & 6 & 3 \\
Mitral regurge & 6 & 3 \\
\hline
\end{tabular}

SWMA indicate systolic wall motion abnormalities; EF, ejection fraction; LV, left ventricle.

Presentation according to the site of myocardial involvement by ECG: ECG data were available for only 201 patients. Of those patients, anterior myocardial involvement was present in $56 \%$, Inferior myocardium involvement in $39.8 \%$. Lateral myocardial involvement in $17.4 \%$. Right ventricular involvement in $2.7 \%$, and posterior myocardial involvement in $2.2 \%$. 


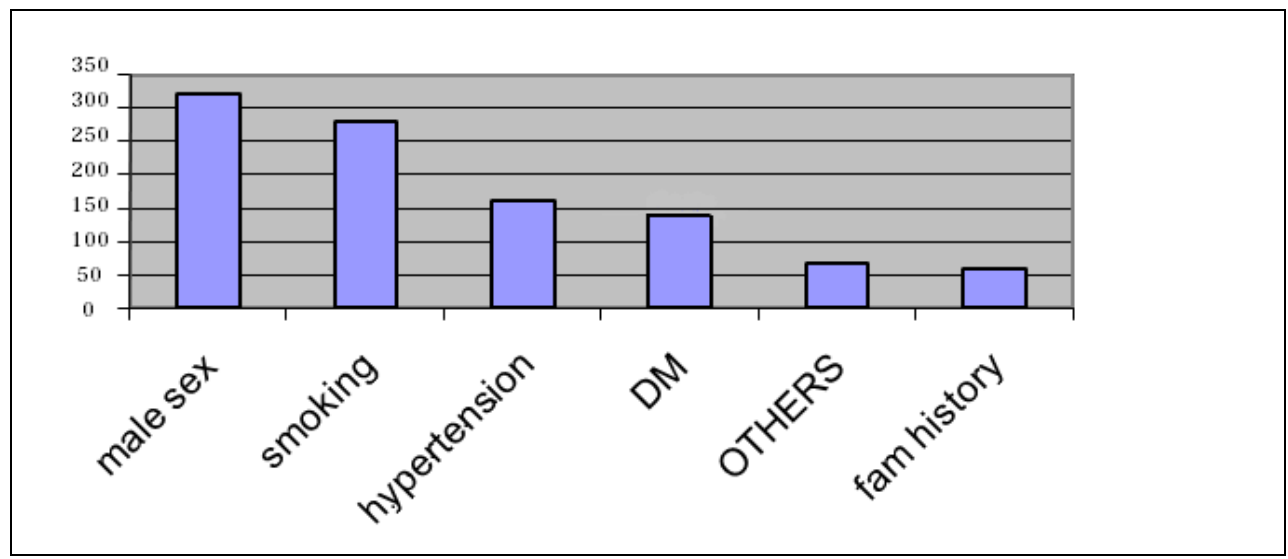

Figure 1: Risk factors of ACS. Others include alcohol,drug abuse and Hashish

Presentation according to ECG and enzymes: More than $2 / 3$ of our population $(70.9 \%, 284$ patients) presented with STEMI. $17.5 \%$ (70 patients) of the study patients presented with NSTEMI.11.8\% (47 patients) of the study patients presented with unstable angina. $1.2 \%$ (5 patients) of the study patients presented with vasospastic angina.

Table 1: Arrhythmias incidence in relation to diagnosis of ACS

\begin{tabular}{|l|l|l|l|l|}
\hline & no & $\%$ & In total & P value \\
\hline STEMI & 182 & $64.1 \%$ & $61 \%$ & $<0.001$ \\
STEMI & 24 & $33.6 \%$ & $8 \%$ & $<0.001$ \\
Unstable angina & 17 & $35.8 \%$ & $5.7 \%$ & $<0.001$ \\
Vasospastic angina & 3 & $60 \%$ & $1 \%$ & 0.520 \\
\hline
\end{tabular}

STEMI indicate ST segment elevation myocardial infarction; NSTEMI, non ST segment elevation myocardial infarction.

Table 3: Arrhythmias incidence in relation to ECG data

\begin{tabular}{|c|c|c|c|c|}
\hline \multirow{2}{*}{\multicolumn{2}{|c|}{ ECG data }} & \multicolumn{2}{|c|}{ Arrhythmias incidence } & \multirow{2}{*}{$P$ value } \\
\hline & & $\%$ & No. & \\
\hline \multirow{2}{*}{ QRS width } & normal & 87 & 163 & $<0.0001$ \\
\hline & wide & 63.2 & 135 & \\
\hline \multirow{2}{*}{ R progressior } & good & 73.8 & 164 & $<0.0001$ \\
\hline & poor & 75.2 & 134 & \\
\hline \multirow{2}{*}{ QT interval } & normal & 71.8 & 212 & $<0.0001$ \\
\hline & long & 82.2 & 86 & \\
\hline
\end{tabular}

\section{Medical treatment}

Routine anti-ischemic treatment in the form of Acetylsalicylate, Clopidogrel, Nitrate and Heparin, were given to most of the study patients $98.3 \%$. The study patients were managed by routine antiischemic treatment as following: Aspirin was given to $98.5 \%$ (394 patients) of the study patients. Clopidogrel was given to 98.8\% (395 patients) of the study patients. Nitroglycerine was given to $97.5 \%$ (390 patients) of the study patients. ACE inhibitors were given to $86.6 \%$ (346 patients). BB was given to $80.3 \%$ ( 323 patients).Statins was given to $95.8 \%$ (383 patients). Heparin was given to $99.9 \%$ (399 patients). Morphia has 
been given to $53.4 \%$ (214 patients). Oxygen has been applied to $22.3 \%$ ( 89 patients). Inotropes has been given as following: Dobutamine has been given to $6.7 \%$, Dopamine has been given to $8.1 \%$, and Adrena- line has been given to $5.5 \%$. Antiarrhythmic drugs were given as following: Amiodarone was given to $11.1 \%$; Lidocaine was given to $3.5 \%$. DC was applied to $10.6 \%$. CPR was done for $6.6 \%$.

Table 4: Relation between arrhythmia and ejection fraction (EF)

\begin{tabular}{|l|c|c|c|c|}
\hline EF\% & No. & arrhythmias & $\%$ & P value \\
\hline$>40 \%$ & 141 & $25 / 141$ & 18.8 & \multirow{2}{*}{0.001} \\
\hline$<40 \%$ & 60 & $38 / 60$ & 63.9 & \\
\hline
\end{tabular}

Echocardiography

ECG data were available for 201 patients. EF was $>40 \%$ in $70.2 \%$ of them. Left ventricular diastolic dysfunction in $58.2 \%$, mitral valve incompetence was found in $2.98 \%$, intracavitary left ventricular thrombus in $2.98 \%$.

\section{ECG characteristics}

As regard $\mathrm{R}$ wave progression, $55.4 \%$ of patients were positive for good $\mathrm{R}$ wave progression. In $46.8 \%$ of patients QRS width was less than or equal 200 ms. Long QT interval was present in $26.2 \%$ of patients.

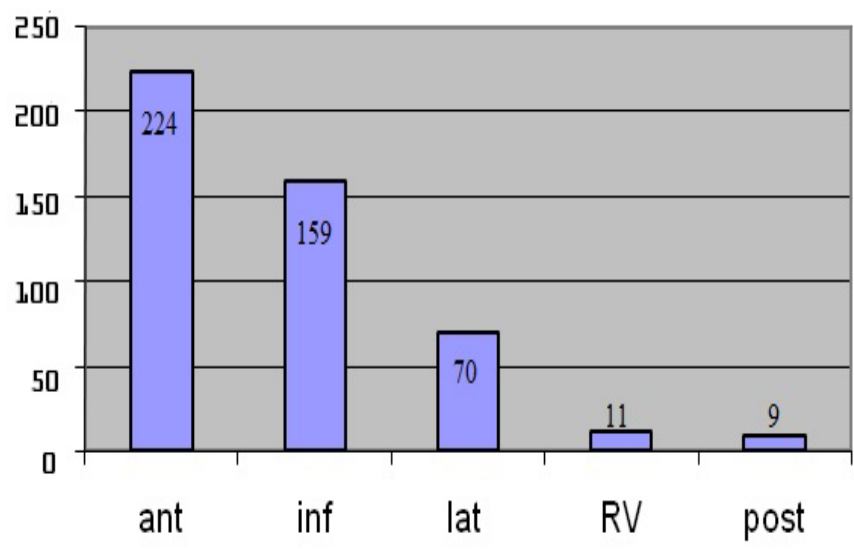

Figure 2: Myocardial involvement incidence in ACS

\section{Incidence and types of arrhythmias}

The incidence of arrhythmias was $74.6 \%$ (298 patients); tachyarrhythmia was found in $57.9 \%$, while brady-arrhythmia was found in $26.4 \%$ of them. The incidence of premature ventricular contraction (PVC) was 39.5\%. Premature atrial extrasystole (PAE) was found in $19.5 \%$. AF in $16.8 \%$. HB in $10.1 \%$. VT in 9.6\%. arrest (asystole) in $8.5 \%$. VF in $8.1 \%$. RBBB in $6.3 \%$, LBBB in $4.3 \%$, and nodal rhythm in $3.1 \%$. This study showed dominance to male gender, $81.8 \%$ i.e. 244 pa- tients of patients with arrhythmias were males, while female gender represented only $18.2 \%$ (54 patients) of patients with arrhythmias. Male gender was the most important risk factor. Hypertensive patients was $74.9 \%$ in this group (122/163 hypertensive patients. They represented $40.9 \%$ of all arrhythmias patients. In obese patients was $67.3 \%$ in this group (65/97of obese patients). They represented $21.8 \%$ of all arrhythmias patients.In diabetic patients was $71.8 \%$ in this group (88/123 diabetic patients); they represented $29.5 \%$ of all arrhythmias patients. 



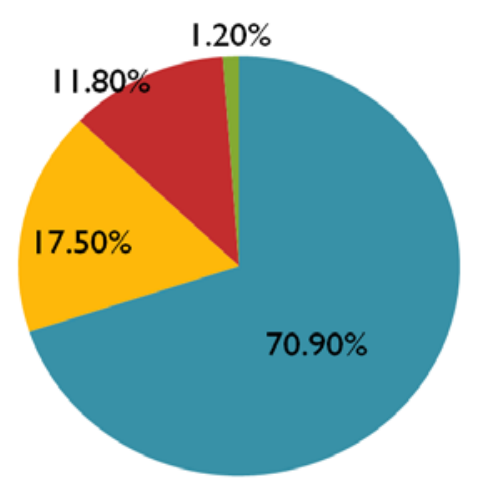

\begin{tabular}{|c|c|}
\hline $\begin{array}{l}\text { STEMI } \\
\text { NSTEMI } \\
\text { UA } \\
\text { VA }\end{array}$ & $\begin{array}{l}\text { STEMI= ST segment } \\
\text { elevation myocardial } \\
\text { infarction } \\
\text { NSTEMI= non ST seg- } \\
\text { ment elevation myocar- } \\
\text { dial infarction } \\
\text { UA= unstable angina } \\
\text { VA=vasospastic }\end{array}$ \\
\hline
\end{tabular}

Figure 3: ACS diagnosis at discharge

The incidence of arrhythmias in STEMI was $64.1 \%$ i.e. 182 out of (284) STEMI patients. In NSTEMI was $33.6 \%$ (24NSTEMI patients. In unstable angina $35.8 \%$ (17/47 UA patients). In vasospastic angina $60 \%$ i.e. 3 patients out of (5) vasospastic angina patients. Arrhythmia was present in $43 \%$ of patients with anterior myocardial involvement i.e. 96 patients out of 224 patients with anterior involvement. $28.9 \%$ patients with inferior myocardial involvement i.e. 46 patients out of 159 patients with inferior involvement. Incidence of arrhythmias in RV involvement was $36 \%$ i.e. 4 patients out of 11 patients with RV involvement.In posterior myocar- dial involvement was 33\% i.e. 3 patients out of 9 patients with posterior involvement. Arrhythmia was present in $91.4 \%$ of patients with past history of IHD (107/117 patients) and was represent $35.8 \%$ of total arrhythmia in the study (107/298 patients). 53\% of patients with past history of $\mathrm{PCl}$ (50/94 patients) and was represent $16.7 \%$ of total arrhythmia in the study i.e. 50 out of 298 patients.35\% of patients with past history of CABG (7/20 patients) and was represent $2.3 \%$ of total arrhythmia in the study (7/298 patients).

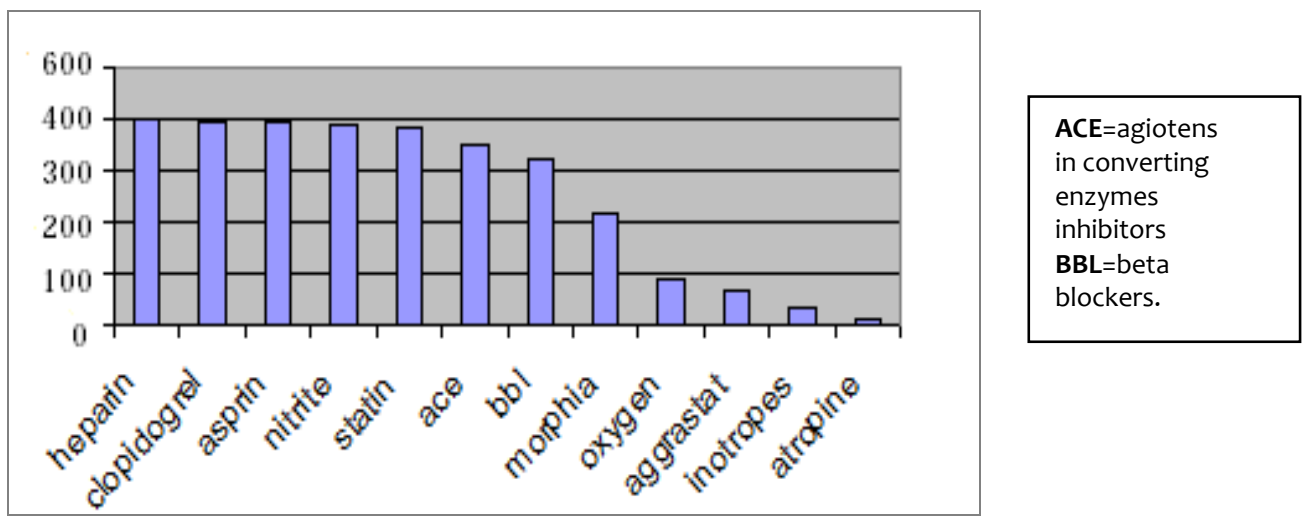

Figure 4: Routine treatment of ACS 


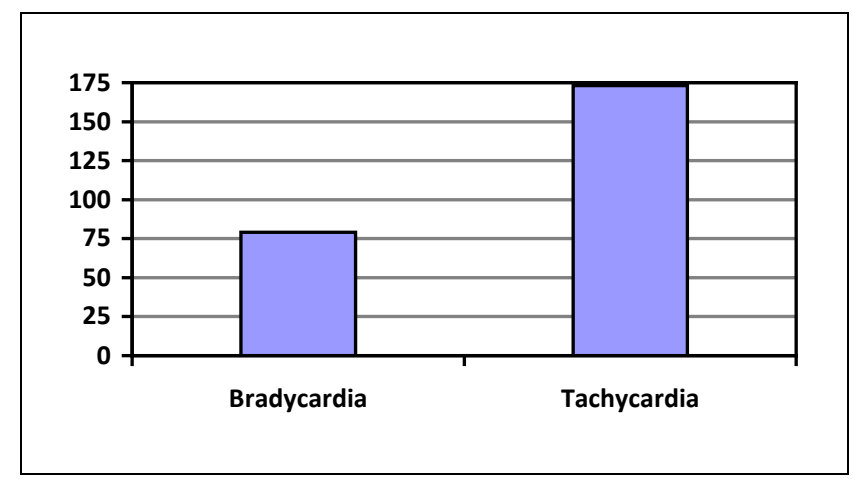

Figure 5:Types of arrhythmias incidence at admission

Arrhythmia was present in 115 out of 154 (74.5\%) patients with past drug history of BB intake and in 115 out of 298 (38.6\%) patients of total arrhythmia in the study. It was also found in $73 \%$ of patients with past drug history of anti-ischemic intake ( 96 out of 131 patients), and in $32.1 \%$ of total arrhythmia in the study (96 out of 298 patients), in $78.3 \%$ of patients with past drug history of OHG intake (26 out of 33 patients), in $8.7 \%$ of total arrhythmia in the study (26 out of 298 patients), in $75.8 \%$ of patients with past drug history of ACEI intake (75 out of 99 patients), in $25.1 \%$ of total arrhythmia in the study (75 out of 298 patients), in $66.4 \%$ of patients with past drug history of insulin intake (12 out of 18 patients), in $4 \%$ of total arrhythmia in the study (12 out of 298 patients). The incidence of arrhythmias in patients with family history of CAD was $76.9 \%$. The incidence of the arrhythmia increased in: i) the presence of the wide QRS (> 2ms) representing $63.2 \%$ in those patients, ii) the presence of poor $\mathrm{R}$ wave progression representing $75.2 \%$ in those patients, and iii) with prolonged QT representing $82.3 \%$ in those patients.

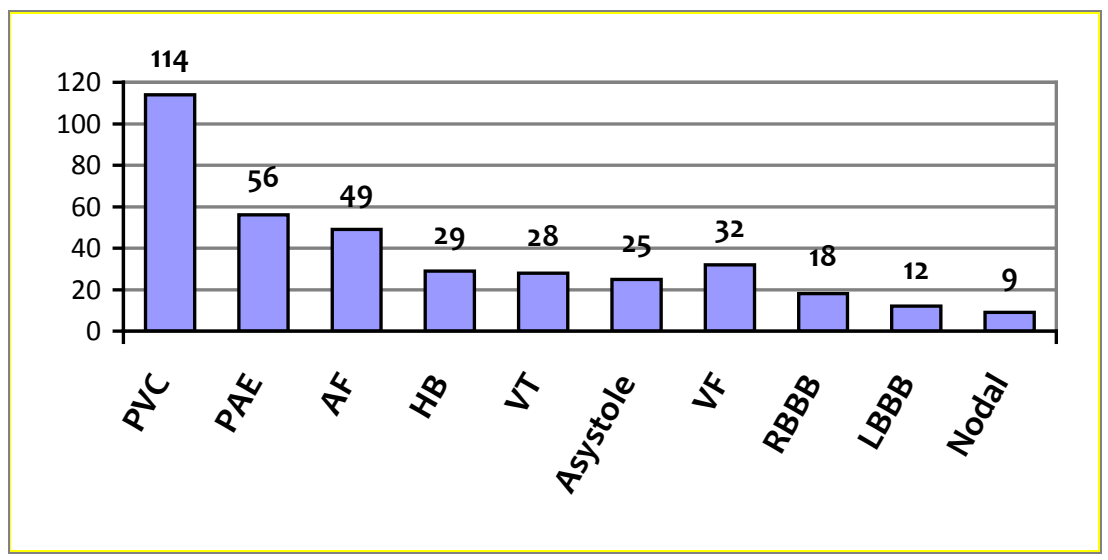

PVC=premature ventricular contraction $\mathrm{PAE}=$ premature atrial extra systole $\mathrm{AF}=$ atrial fibrillation $\mathbf{H B}=$ heart block VT= ventricular tachycardia

$\mathbf{V F}=$ ventricular fibrilation LBBB=left bundle branch block

RBBB=right bundle branch block.

Figure 6: Incidence of differernt types of arrhythmias at admission 


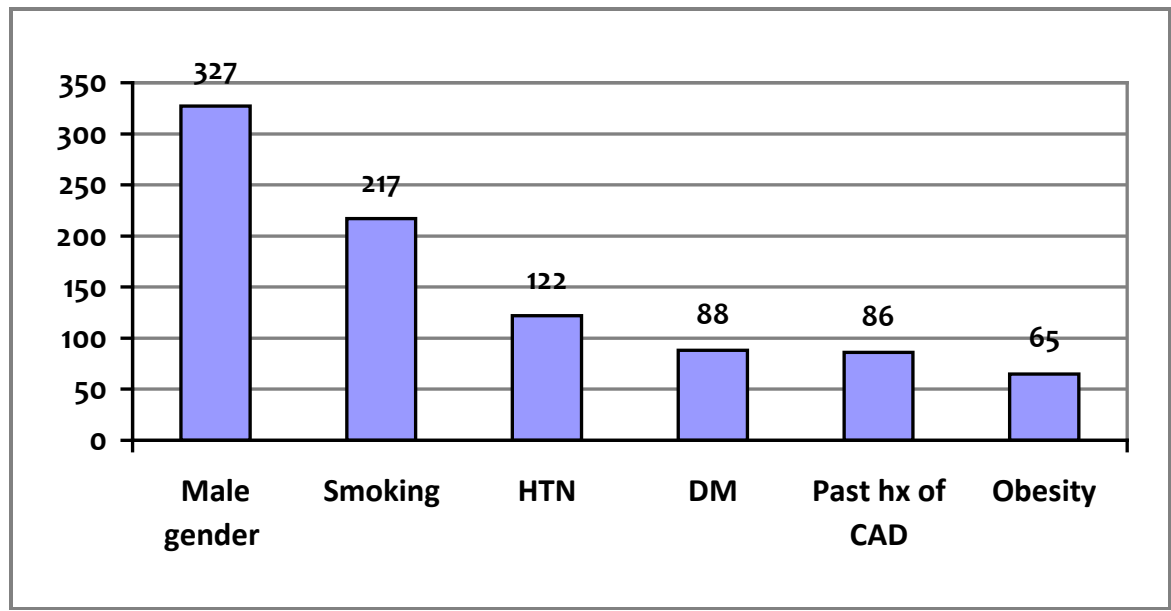

$\mathbf{H T N}=$ hypertension DM=diabetes mellitus CAD=coronary artery diseases.

Figure 7: Risk factors of arrhythmias in ACS

As regards arrhythmia in relation to $E F$, we found that the less the EF the more the incidence of arrhythmias. There was a highly significant relation between cardiac systolic performance (EF\%) and occurrence of arrhythmias, as the incidence of arrhythmias was $63.9 \%$ among patients with $\mathrm{EF}<40 \%$, while it was $18.8 \%$ among patients with normal EF. In hospital morbidity was estimated during in-hospital stay to be $29.7 \%$ (119 patients). Left ventricular dysfunction occurred in $16.9 \%$ (68 patients). Arrhythmic complication (persistent arrhythmia) oc- curred in $11.3 \%$ (45 patients). Renal impairment occurred in $2 \%$ ( 8 patients).Persistent ischemia i.e. patients for urgent CABG were $5.3 \%$ (21 patients). Ischemic mitral regurgitation occurred in $1.8 \%$ i.e.7 patients. Ventricular septal rupture (VSR) occurred in 2 patients (0.5\%). By dividing the study patients into three subgroups according to the diagnosis, morbidity incidence was highest in STEMI (25.6\%) followed by non STEMI (15.6\%), and lastly unstable angina (9.4\%), while vasospastic angina did not show any morbidity.

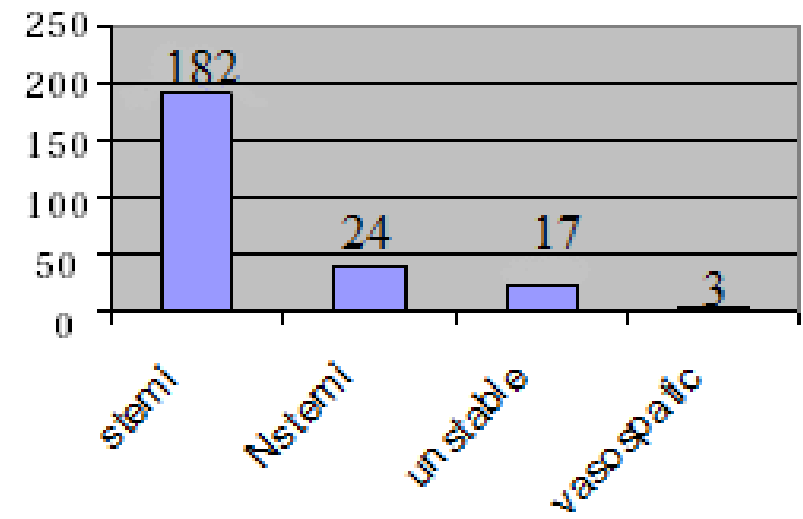

Figure 8: Incidence of arrhythmias in relation to diagnosis 


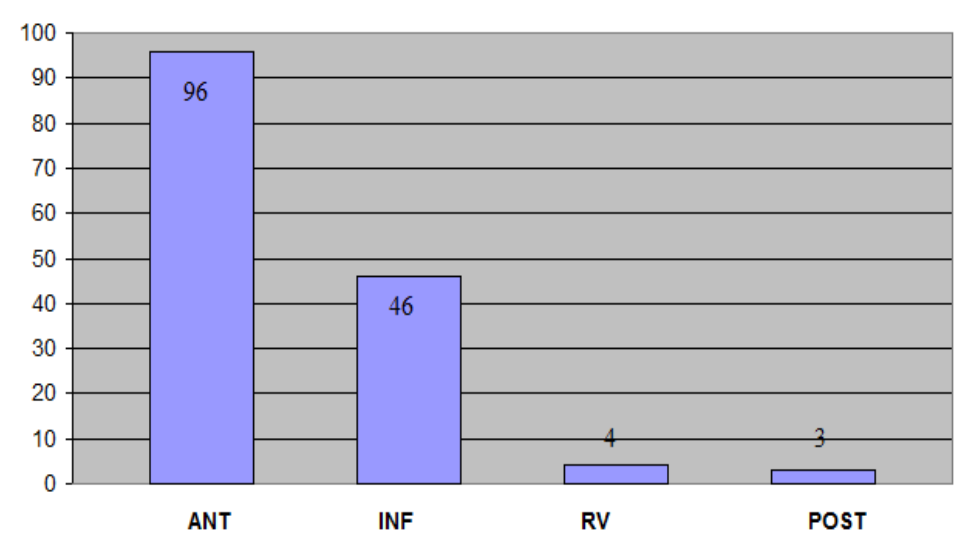

Ant $=$ anterior wall Inf= inferior wall

Lat= lateral wall

Post $=$ posterior wall

$\mathbf{R V}=$ right ventricle

Figure 9: Incidence of arrhythmias in relation to ECG distribution

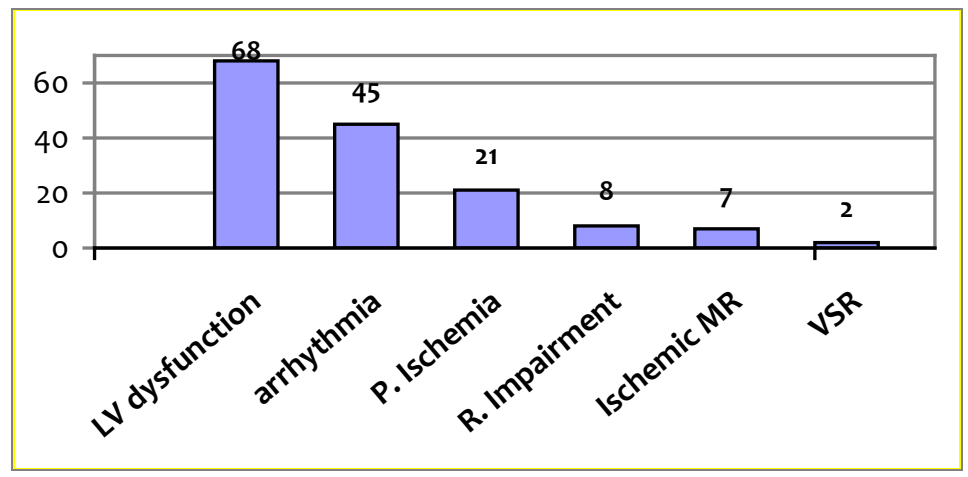

$\mathbf{L V}=$ left ventricle

$M R=$ mitral regurge

VSR=ventricular septal

rupture.

$\mathbf{P}=$ persistant

$\mathbf{R}=$ renal

Figure 10: Morbidity in ACS

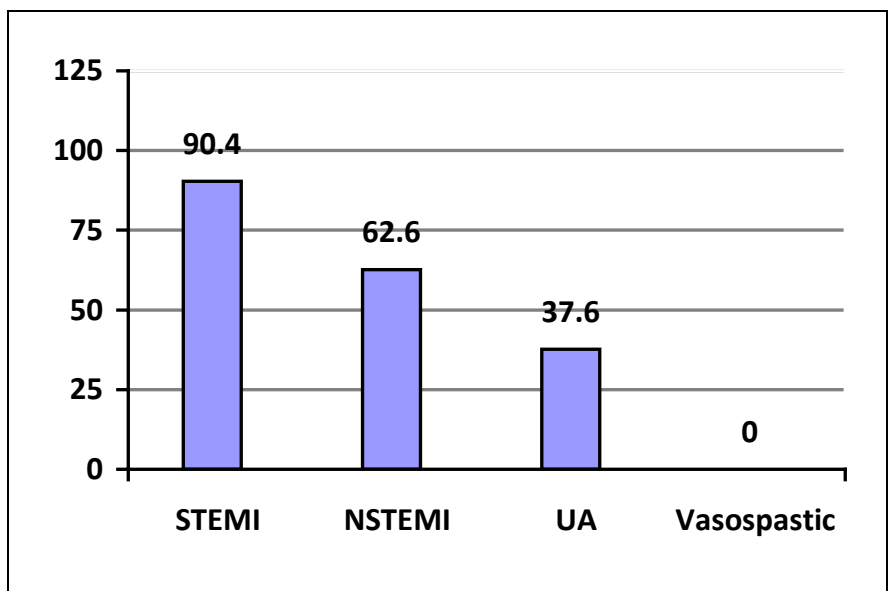

STEMI=ST segment elevation myocardial infarction NSTEMI=non ST segment elevation myocardial infarction

UA=unstable angina.

Figure 11: Morbidity in relation to ACS diagnosis 

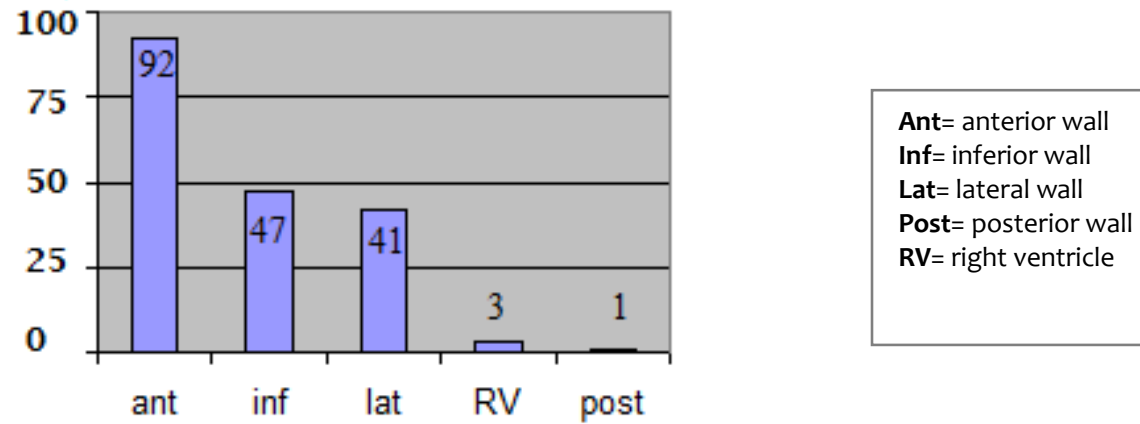

Figure 12: Morbidity in relation to myocardial infarction site.

The study patients were divided into different groups according to myocardial involvement. Higher morbidity incidence was observed in $92(77.7 \%)$ patients with anterior myocardial involvement, followed by inferior myocardial involvement in 47 patients (39.4\%) of the total morbidity, followed by lateral myocardial involvement in 41 patients (34.8\%) of the total morbidity and lastly right ventricular involvement was seen in 3 patients and posterior myocardial involvement in one patients; both represented $3.4 \%$ of the total morbidity of the study patients. The study patients were divided into different groups according to their past history of medical disease. The highest morbidity incidence was present in diabetic patients (49 patients, $41.6 \%$ ) of the total morbidity, followed by hypertensive patients (48 patients, 40.3\%), and lastly ischemic patients (46 patients,.38.6\%). Smoking represented the prevalent risk factor for increased morbidity incidence in the study patients; they were 78 patients and 52 patients with past history of ischemic heart disease, 31 patients obese and 22 patients with positive family history. In hospital mortality was estimated to be $4.1 \%$ (16 patients), and by dividing the study patients into subgroups, it revealed that mortality was more prevalent in STEMI group was (12 patients, $4.4 \%$ ), while in NSTEMI group It was seen in (4 patients, $5.7 \%$ ). No moralities had occurred in unstable angina. Male gender showed the highest incidence of mortality (14 patients, $84.8 \%$ )of the total mortality incidence and $4.5 \%$ mortality incidence of the male gender group. Smoking was associated with $75.8 \%$ of the total mortality incidence and $4.5 \%$ of the smoking population group. DM was associated with a mortality incidence of $45.5 \%$ of the total mortality incidence and $5.8 \%$ of the diabetic population group.

\section{Discussion}

Arrhythmia is very common in ACS, and shares a lot in both its morbidity and mortality. Development of ventricular arrhythmias (VAs) in the setting of an acute myocardial infarction (MI) is one of the most frequent causes of death. However, improvements in arrhythmia detection and treatment have a major impact on outcomes from VAs associated with acute $\mathrm{MI}$, resulting in improved hospital prognosis ${ }^{(5)}$. The objectives of this observational study were to describe the incidence and clinical outcomes of arrhythmias in patients hospitalized with an acute coronary syndrome. Also to identify patient characteristics associated with an increased risk for developing arrhythmias for purposes of directing targeted surveillance and/or treatment efforts toward high-risk patients. The study of such data in our region may demonstrate the proper risk profile of our patients both for ACS and those with arrhythmias associated with ACS and compare it with those 
of the European or American standards whose guidelines are currently applied in Egypt as well as most of the Middle East countries. The availability of such data could be an appropriate database allowing the initiation of national guidelines concerning ACS, increase our attention to a very big deal of morbidity and mortality which is arrhythmias. However in Egypt, no registries were available till now despite the overwhelming increase in the number of ischemic heart disease patients and the change in the natural history of the disease affecting certain classes of patients particularly young age and women in addition to the aggressive nature in many patients ${ }^{(6)}$. We analysed data from our registry of patients with ACS from Suez Canal university hospital in Ismailia, so is more likely to reflect the present scenario of ACS in Egypt. The current registry enrolled 400 patients with acute coronary syndrome.We found Arrhythmia was present in $74.6 \%$ of study population, $57.6 \%$ of them were tachyarrhythmia while bradyarrhythmia was $26.4 \%$. There was increased arrhythmia incidence in STEMI $64.1 \%$, anterior myocardial involvement $43 \%$. Male gender and smoking were the strongest risk factors of arrhythmias.The incidence of different types of arrhythmia was in the following order : $39.5 \%$ PVC, $19.5 \%$ PAE, $16.8 \% \mathrm{AF}$, and $10.1 \% \mathrm{HB}, 9.6 \% \mathrm{VT}, 8.5 \%$ asystole, 8.1\% VF, $6.3 \%$ RBBB, $4.3 \%$ LBBB and $3.1 \%$ Nodal rhythm. In our study incidence of arrhythmia was in the following order: AF (16.8\%), and HB (10.1\%), VT (9.6\%), asystole $(8.5 \%)$ and VF (8.1\%). In other studies, overall serious arrhythmic complication in TACSR (Thailand Acute Coronary Syndrome Registry) ${ }^{(7)}$ defined as second and/or third degrees AV block, VT and VF) was $16.6 \%$, while the incidence of both VT/VF and AV Block in GRACE (Global Registry of Acute Coronary Events) ${ }^{(8)}$ was $7.7 \%$. In the current study the risk profile of arrhythmias in ACS Included: male gender as the most prevalent risk factor for arrhythmias incidence $(81.8 \%)$, followed by smoking $72.8 \%$, hypertension (40.9\%), DM (29.5\%), and past history of IHD (35.8\%). Hashish and drug abuse represented (17.7\%). In TACSR Congestive heart failure within the first 48 hours, current use of tobacco and cardiac troponin elevation were associated with significantly higher arrhythmic complications during hospitalization, also smoking history was associated with an increased risk for ventricular arrhythmias ${ }^{(7)}$. Whereas in GRACE, prior myocardial infarction and percutaneous coronary intervention were associated with a reduced risk $^{(8)}$. It was noticed that there was low incidence of arrhythmias in those patients on current antiischemic treatment, and in those who had done previous $\mathrm{PCl}$. This may reflect that ischemia management is protective against arrhythmias. Arrhythmias were more prevalent in those patients with positive history of sweating, nausea, vomiting, and typical chest pain with radiation and was higher in those who had syncope, cardiogenic shock and lung congestion. In other studies, GRACE, GULF RACE and SPACE (Saudi Project for Assessment of Acute Coronary Syndrome $)^{(9)}$, cardiogenic shock and decreased LV function (Killip classification) were associated with increased incidence of ventricular arrhythmias.

There was a possible association of increased arrhythmias incidence and B-blocker intake in spite of its known role in managing arrhythmias. This may be explained by its being associated with arrhythmogenic factors like bad cardiac condition and abuse of Bblocker i.e. intermittent intake and rebound effect. The incidence of arrhythmias is increased in STEMI it was $64.1 \%$, followed by NSTEMI $33.6 \%$ and UA $35.8 \%$. Serious ventricular arrhythmias (Sustained VT/VF) were present in $4 \%$ of STEMI, and $2 \%$ each for NSTEMI and UA. For ECG distribution the incidence of arrhythmias was increased in 
Anterior $\mathrm{MI}$, followed by Inferior $\mathrm{MI}$, and lastly both RV and posterior infarction. In GRACE and SPACE registries there was increased incidence of ventricular arrhythmias in those of ST deviation either STEMI or NSTEMI. In GRACE study Sustained VT/VF occurred in $10 \%$ of STEMI, $4 \%$ of NSTEMI and $2 \%$ of UA, while in TACSR patients ventricular arrhythmia was more prevalent with $19.4 \%, 8.1 \%$ and $1.8 \%$ respectively ${ }^{(7,8)}$.

Regarding the initial presentation, our study patients were diagnosed as $70.9 \%$ STEMI and $17.5 \%$ NSTEMI, and $11.8 \%$ Unstable Angina. In GRACE there was 39\% had STelevation myocardial infarction, 39\% had non-ST-elevation infarction, and $22 \%$ had unstable angina.While in GULF RACE ${ }^{(10)}$ STsegment elevation myocardial infarction was present in $37 \%$, non-ST-segment elevation myocardial infarction in 32\%, and unstable angina in $29 \%$ In EHS (EURO heart survey) ${ }^{(11)}$, NST-ACS patients comprised $48 \%$ of the patients, $47 \%$ had an initial diagnosis of ACS with STEMI, whereas five percent of the patients presented with an undetermined (ECG) pattern.

The mean age was 54 years, which is far less than other international and national registries. In GRACE it was 64.9 years, in GULF RACE it was 56 years in average; however it was higher in residents than in expatriates and in EHS where the mean age of patients was 64.7 years. This means that in Egypt, ischemic heart disease occurs at an earlier age causing a great economic burden on personal and public levels. This study showed dominance of male gender, it represented $79.2 \%$ of the study patients. Our result was similar to other studies. Male gender was $65 \%$ to $73 \%$ in the GRACE, $70.1 \%$ in EHS and $76 \%$ in GULF RACE registry. $69.8 \%$ of the study patients were current smokers. This was the highest incidence of smoking ever met in comparison to other studies, in GRACE it ranged from $24 \%$ to $42 \%$. In GULF RACE it was
$36.6 \%$, however in expatriates it was higher reached, while in EHS it represented 36.5\%. $40.8 \%$ of the study patients were known hypertensive. In GRACE it ranged from 33\% to $51 \%, 57 \%$ in EHS and $49 \%$ in GULF RACE. In GULF RACE DM was found in $50.2 \%$, in our study it was $38.9 \%$. While In GRACE it ranged from 10 to $17 \%$. In our study $24.4 \%$ of patients were obese. In GRACE $42 \%$ were overweight, $25 \%$ were obese, $6 \%$ were morbid obesity, while in EHS 32.8\%\% were obese and $53 \%$ centrally obese. In GULF RACE obesity was in $29 \%$ among those with normal glucose tolerance, and 55\% among those with previously unknown diabetes. $14.6 \%$ of the study patients had positive family history of CAD. In TACSR it was present in $9.3 \%$ of their patients, while in GULF RACE it was $16 \%$ in ACS patients $\leq 40$ years of age compared with $7 \%$ in patients $>40$ years of age. Family history of premature coronary artery disease was present in $11 \%$ of SPACE registry patients.

In this study the patients who had taken Aspirin represented 98.5\%, while in GRACE and EHS it was $94 \%$ to $95 \%$, clopidogrel represented $98.8 \%$ while in GRACE and EHS it was $70 \%$, to $85 \%$ those who has taken ACE inhibitors was $86.6 \%$, it was in GRACE and EHS $65 \%$ to $80 \%, \mathrm{BB}$ in the study represented $80.3 \%$ while in GRACE and EHS it was $70 \%$ to $90 \%$, in our study Statins represented $95.8 \%$, while in GRACE and EHS it was $85 \%$ to $90 \%$ Heparin was given to $99.9 \%$ of the study patients. This reflected the good adherence to guideline treatment. There was $29.7 \%$ morbidity in the study patients about $77.7 \%$ of them were STEMI patients, it was mostly of anterior myocardial involvement. The in hospital mortality in our patients was estimated to be $4.1 \%$ as average allover study, $75.8 \%$ of total mortality was in the STEMI patients. In GRACE there was also increased mortality in STEMI patients and total mortality ranged from $4 \%$ to $6.6 \%$, while it was $4 \%$ in 
$\mathrm{EHS}^{(11)}$. So mortality rate does not differ a lot from other developed nations. In TACSR In-hospital mortality for sustained VT/VF was $48 \%$ and for AV block was $31 \%$. lar study in the United States ${ }^{(13)}$, The Worcester heart attack study, showed that complete AV block was also associated with a significant mortality of $46.8 \%$. Pooled data from various studies has shown that 60 days mortality varied from $20-65 \%$, depending on the presences of $\mathrm{VT}, \mathrm{VF}$, or both . There have been further analyses of demographic factors, management, investigation and patient out come to make risk assessment evaluation of morbidity and mortality either in GRACE or GULF RACE aiming for secondary prevention and targeted surveillance. In GULF RACE, SPACE and TACSR there was increased mortality incidence in those with positive incidence of ventricular arrhythmias either those who were STEMI or NSTEMI ${ }^{(7,9,10)}$ In the current study, $84.8 \%$ of the total mortality were males, $75.8 \%$ of the total mortality were current smokers, $54.5 \%$ of total mortality were diabetic. Previously, STEMI represented $75.8 \%$ of total mortality and $70.1 \%$ of total morbidity patients. $75.8 \%$ of total mortality were anterior MI patients ${ }^{(12)}$.

\section{Conclusion}

The study helped to show our demographic characteristics, risk factors for both ACS and those who had arrhythmias. The patients were younger, smokers, suffering mainly of hypertension, they were more prone to arrhythmia incidence, because they were smokers, mostly STEMI, and of anterior myocardial involvement. The study helped to show that the management was so adherent to guidelines, but deficient for proper arrhythmias management

\section{References}

1. Zellweger MJ, Schaer BA, Cron TA, Pfisterer ME, Osswald S. Elevated troponin levels in absence of coronary artery disease after supraventricular tachycardia. Swiss Med Wkly.2003;133(31-32):439-441.

2. Bacaner $M$, Dembo DH. Arrhythmia and Acute Coronary Syndrome Suppression and Cardiac Resuscitation Management with Bretylium. Am J Ther 2009, 16(6): 534-542.

3. Granger CB, Goldberg RJ, Dabbous O, Pieper KS, Eagle KA, Cannon CP, Van De Werf F, Avezum A, Goodman SG, Flather MD, Fox KA; Global Registry of Acute Coronary Events Investigators. Predictors of hospital mortality in the Global Registry of Acute Coronary Events. Arch Intern Med 2003;163 (19):2345-2353.

4. Lombardi F. Timing of arrhythmic death after myocardial infarction, does it affect timing of ICD implantation, Eur Heart $J$ 2005; 26 (14), 1350-1352

5. Shah M1, Akar FG, Tomaselli GF.Molecular Basis of Arrhythmias. Circulation 2005;112 (16):2517-2529.

6. Bassand JP, Priori S, Tendera M. Evidencebased vs. 'impressionist' medicine: how best to implement guidelines, Eur Heart $J$ 2005; 26 (12), 1155-1158.

7. Ngarmukos $T$, Sriratanasthavorn C, Sansaneevithayakul B, Kasemsuwan P, Angkasuwapala K. Cardiac arrhythmias in Thai Acute Coronary Syndrome Registry. j Med Assoc thai , 2007 ;90(11):58-64.

8. Fox KA, Eagle KA, Gore JM, Steg PG, Anderson FA; GRACE and GRACE2 Investigators. The Global Registry of Acute Coronary Events, 1999 to 2009--GRACE., Heart 2010; 96(14):1095-1101.

9. AlHabib KF, Hersi $A$, AlFaleh $H$, Kurdi $M$, Arafah $M$, Youssef $M$, AlNemer K, Bakheet A, AlQarni A, Soomro T, Taraben A, Malik A, Ahmed WH. The Saudi Project for Assessment of Coronary Events (SPACE) registry: Design and results of a phase 1 pilot study, Can J Cardiol. 2009; 25(7): e255-e258.

10. Zubaid MB. Preliminary results from gulf registry of acute coronary events (GULF RACE). Heart views 2008, volume 8: 155158.

11. Michel K, Helmut D. Lessons from the European Heart Survey, Circulation 2006, $113: f 25-\mathrm{f} 28$. 
12. Access Investigators. Management of Acute Coronary Syndromes in Developing Countries : Acute Coronary Events-A Multinational Survey of Current Management strategies. Am Heart J, 2011; 162(5):852-859.

13. Spencer FA, Jabbour $S$, Lessard $D$, Yarzebski J, Ravid S, Zaleskas V, Hyder M, Gore JM, Goldberg RJ. Two-decade-long trends (1975-1997) in the incidence, hospitalization, and long-term death rates associated with complete heart block complicating acute myocardial infarction: a community-wide perspective. Am Heart J. 2003;145(3):500-507. 